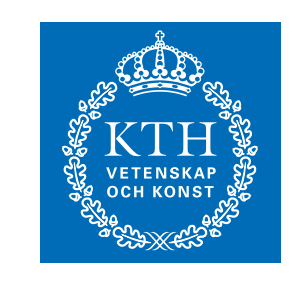

KTH Electrical Engineering

\title{
On Performance Trade-Offs in Cognitive Networks
}

C2010 IEEE. Personal use of this material is permitted. However, permission to reprint/republish this material for advertising or promotional purposes or for creating new collective works for resale or redistribution to servers or lists, or to reuse any copyrighted component of this work in other works must be obtained from the IEEE.

\section{E. STATHAKIS, N. SCHRAMMAR, L. K. RASMUSSEN, AND M. SKOGLUND}

Stockholm 2010
Communication Theory Department
School of Electrical Engineering
KTH Royal Institute of Technology




\title{
On Performance Trade-Offs in Cognitive Networks
}

\author{
E. Stathakis*, N. Schrammar, L. K. Rasmussen, and M. Skoglund \\ School of Electrical Engineering, KTH, Royal Institute of Technology, Stockholm, Sweden \\ Email: $\{$ eftsta,nisc,lkra,skoglund\}@kth.se
}

\section{INTRODUCTION}

During the last few years, we have witnessed a tremendous increase in the demand for new wireless applications and services. The demand for high data rates and ubiquitous coverage suggests that emerging wireless systems should operate within flexible standardization regulations, and the cognitive radio paradigm is within this scope. Cognitive radio systems are motivated by the need for flexible spectrum assignment policies. Their operation targets efficient utilization of licensed and unlicensed spectrum by joint and adaptive coordination of their decisions and transmission strategies. One example are TV white spaces (TVWS) which corresponds to frequency bands in the VHF/UHF bands released by the transition from analog to digital TV. Special network architectures, like femtocells would also be possible candidates for cognitive operation.

Our approach here, involves the consideration of underlay cognitive networks, where primary and cognitive users coexist but cooperate only with terminals of their class. Information-theoretic analysis of such networks involves, in principle, the consideration of general multiterminal networks for which the capacity region is not known in general. In this paper we employ the recently proposed deterministic framework [1] to model and analyze cognitive networks. Despite its simplicity, this model captures fundamental aspects of networks and allows explicit calculation of the capacity of multicast relay networks. The performalnce results we obtain and the conclusions that we draw in the deterministic field can provide uscful insights that can be applicd on the analysis of Gaussian networks.

\section{Problem Formulation}

Cognitive operation can be identified in various scenarios. In this paper we shall investigate performance trade-offs in the downlink of a licensed system, which could be a DVB system realized as shown in Fig. 1. Primary transmission is assisted by relays, randomly and uniformly distributed over the cell. Each primary user (PU) is allowed to select and occupy limited networks resources (relays) for its transmission. A secondary network, with own architecture, is operating within a small designated area, located inside the primary cell. Moreover, the layout consists of a square cell where the primary BS is located on the plane root. We assume a pathloss model with attenuation exponent $a=2$, i.e., the received power decays with the square of the distance, as $P \propto \frac{1}{d^{2}}$.

Our purpose is to evaluate the effect of specific parameters in the network. For example, the total number of available relays in the cell, will affect individual rates because each PU will have the chance to forward its message through relays subject to different levels of interference. Moreover, the degree to which the primary system is cognitive-aware might also influence performance, since PUs that are unaware/aware of the sccondary users (SUs) can employ different criteria, SNR- or SINR-based, in their relay selection process. One viewpoint that has also received attention, concerns 'protection' of the primary system. In this case, the SUs should maintain a minimum distance (guard zone) from each primary receiver to avoid injecting and receiving, respectively, severe interference.

The authors would like to thank the European Union for providing partial funding of this work through the EU FP7 project INFSO-ICT-248303 QUASAR. 


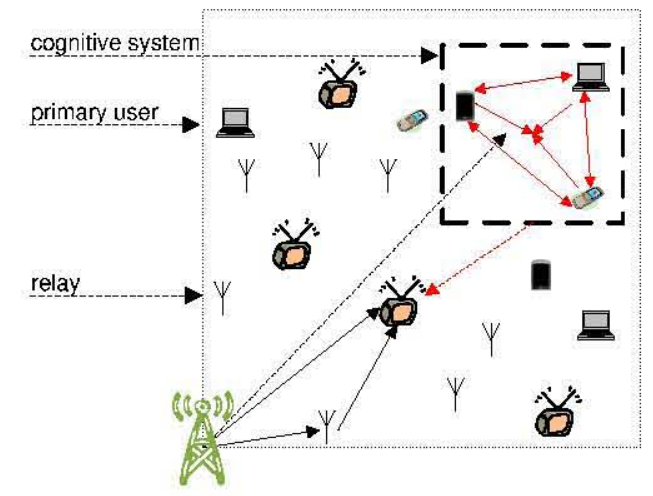

Fig. 1. Downlink operation of primary and secondary ad-hoc systems. Solid lines correspond to own system transmission, dotted lines to the interference.

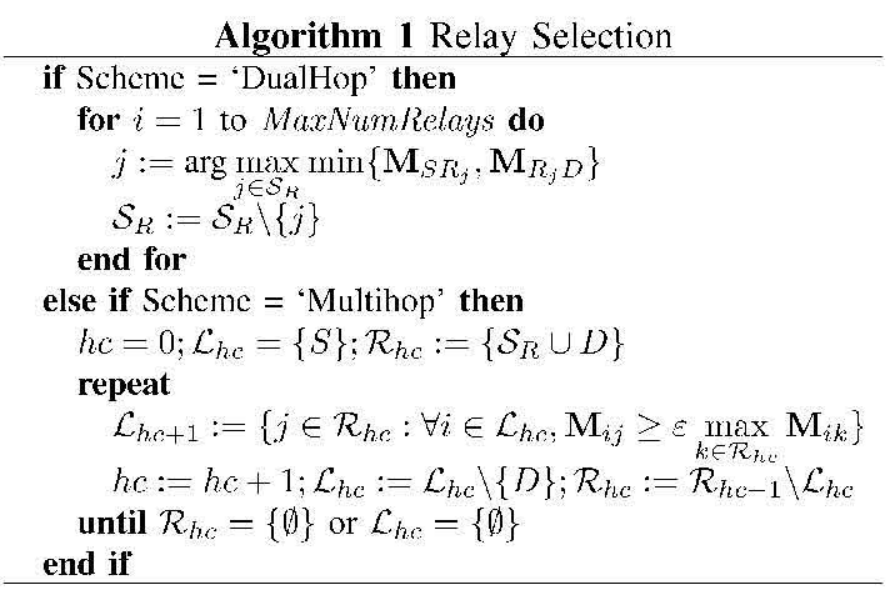

\section{The Deterministic MOdel}

The scenario under consideration falls into the class of general multiterminal networks (GMN). Establishing the capacity region of such networks, is a longstanding open problem in information theory. The design of transmission strategies that achieve capacity region in GMN is a challenging problem, and even in commonly studied Gaussian networks optimal solutions do not exist, in general. However this issue has been resolved for deterministic models, which we will briefly introduce next.

\section{A. Model Description}

In the deterministic framework, we essentially discard the stochastic nature of the network and assume deterministic channel transition functions, i.e., $p(\cdot \mid \cdot) \in\{0,1\}$. In order to familiarize the reader and make the analysis comprehensive, we shall illustrate the fundamental principles of the deterministic framework.

Consider a Gaussian network with $M$ terminals. Let $x_{i}$ and $y_{j}$ denote the transmitted and received signals at nodes $i$ and $j$, respectively. Let $h_{i j}$ be the channel gain from node $i$ to node $j$ and $z_{j} \sim \mathcal{N}(0,1)$ be the noise at receiver $j$. Then, the received signal at the $j$-th receiver can be expressed as

$$
y_{j}=\sum_{i=1, i \neq j}^{M} h_{i j} x_{i}+z_{j}, \quad j=1, \ldots, M .
$$

Note here, that the transmit signal obeys an input power constraint $\mathrm{E}\left[\left|x_{i}\right|^{2}\right]=1$. Under these premises, the signal-to-noise ratio can be expressed as $\mathrm{SNR}_{i j}=\left|h_{i j}\right|^{2} \forall i, j \in V, i \neq j$. According to [2], we can associate each channel gain (equivalently SNR) with an integer $n_{i j}=\left\lceil\frac{1}{2} \log _{2}\left(\mathrm{SNR}_{i j}\right)\right\rceil^{+}$, which represents the bits that go through the channel intact. Here, the factor $\frac{1}{2}$ holds for real random variables, hence for their complex equivalents we should instead use 1. Then, according to [2] we can approximate (1), in the high SNR region, as

$$
\mathbf{y}_{j}=\sum_{i=1, i \neq j}^{M} \mathbf{S}^{q-n_{i j}} \mathbf{x}_{i}, \quad j=1, \ldots, N,
$$

where $\mathbf{x}_{i} \in \mathbb{F}_{2}^{q}$ is a vector containing the binary expansion of the input signal $x_{i}, q=\max \left\{n_{i j}\right\}$ and $\mathbf{S}$ is a shift matrix that shifts $\mathbf{x}_{i}$ by $\left(q-n_{i j}\right)$ bits. Note that additions in (2) are modulo-2, hence there is no carry-over produced from the summation and the model is termed linear finite field. An elaborate discussion of these concepts can be found in [1], [2].

Now consider an arbitrary relay network $V$ with a source $S$ and a set of destinations $\mathcal{D}$. Application of this approach leads to noise elimination and approximation of the initial network with a deterministic one. For such types of network models it has been shown that we can achieve the corresponding cut-set bound using random coding arguments. The main results concerning the linear finite field deterministic model (LFFDM) are summarized in the following two theorems [1]. 
Theorem 1: The capacity of the relay nctwork in the LFFDM is $C_{\mathrm{det}}^{\mathrm{uni}}=\min _{\Omega \in \Lambda_{\Gamma}}$ rank $\mathbf{G}_{\Omega, \Omega^{c}}$.

Theorem 2: The capacily of the relay network in the LFFDM with one message and multiple destinations $D \in \mathcal{D}$ is $C_{\mathrm{dct}}^{\text {multi }}=\min _{D \in \mathcal{D}} \min _{\Omega \in \Lambda_{D}} \operatorname{rank} \mathbf{G}_{\Omega, \Omega^{\circ}}$, where $\Lambda_{D}=\left\{\Omega: S \in \Omega, D \in \Omega^{c}\right\}$ and $\mathbf{G}_{\Omega, \Omega^{\circ}}$ denotes the transfer matrix from set $\Omega$ to set $\Omega^{c}$. To be more precise $\mathbf{G}_{\Omega, \Omega^{\circ}}$ is the super matrix whose input/output vector is formed by the juxtaposition of the binary input/output vectors $\left(\mathbf{x}_{i}, \mathbf{y}_{j}\right)$ of $\Omega / \Omega \Omega^{c}$ and each input-output pair $(i, j)$ is associated with its shift matrix $\mathbf{S}^{q-n_{i j}}$ in $\mathbf{G}_{\Omega, \Omega^{\circ}}$.

These two theorems state that in deterministic relay networks, with one transmitted message, we can achieve the cut-set bound.

\section{B. Gaussian vs. Deterministic Model}

Prior to proceeding to the next sections, we shall swiftly describe some advantages of the deterministic framework compared to its Gaussian equivalent. It will become obvious, later, that relay selection is less involved in the LFFDM. Selection methods in Gaussian settings essentially require optimizing the power allocation across all users, rendering the problem computationally intractable. Moreover in dualhop Gaussian relay networks there is no universal strategy for optimal results; one usually considers a combination of decode-and-forward and compress-and-forward strategies, depending on relay location.

Even though in a network $V$ we have $2^{|V|}$ cut-sets, the authors in [3] devised an algorithm to calculate the LFFDM capacity in polynomial time. Further research in this field shows that analysis in the LFFDM can lead to significant insights, which can be translated into cfficient stratcgics in the Gaussian model, see [4], [5]. In [6] it was shown that, despite the lack of direct connection between the two models, the LFFDM is still a good approximation of the Gaussian model.

\section{Relay Selection}

In this section we shall briefly describe the relay selection strategies for dual and multihop transmission. The deterministic framework allows for simple selection strategies. Our proposed algorithm for multihop scenarios implements a simple but efficient and parameterized solution, avoiding brute-force search. The multihop algorithm is not hop-constrained but on the contrary is trying to utilize all available resources.

In the description of algorithm 1 on page $2, S$ denotes the source, $\mathcal{S}_{R}$ the set of relays and $D$ the destination. For the dual-hop case MaxNumRelays is the maximum number of relays that the source may occupy. In the multihop scenario $h c$ is the hop counter, $\mathcal{L}_{b c}$ is the set of nodes in the $h c$-th layer and $\mathcal{R}_{h c}$ is the set of remaining relays after the $h c$-th assignment. $\mathbf{M}$ is the metric that measures the quality of each link in terms of SNR or SINR, depending on the level of system interference awareness, and is employed as a selection criterion. Finally $\varepsilon \in(0,1]$ is a system defined parameter, so that in each hop a node gets assigned not only the relay that maximizes the metric $M$ but also those whose metric is $\varepsilon$-close to the relay maximizing the metric. A value of $\varepsilon$ close to one makes the system 'more multihop' whereas a value close to zero makes the system essentially dual-hop.

\section{Scenarioand Numerical Results}

In this section we present the scenario under investigation, along with numerical results. We assume a wireless cognitive mesh network where the source and the sink are located in opposite locations inside the cognitive area. An arbitrary number of intermediate sccondary nodes, whose selection is based on the multihop selection algorithm presented above with $\varepsilon=0.8$, act as relays assisting transmission. The primary system is a relay network with at most one relay at its disposal. This choice serves the visualization of the results in terms of primary system SINR, since in more sophisticated network architectures it is difficult to define a global SINR measure, against which to express capacity. Fig. 2 and Fig. 3 illustrate the effect of these parameters on system performance.

In Fig. 2 we see the effect of relays and system awareness. PU awareness can provide substantial gains in low SINR, where a priori knowledge of interference is crucial and leads to smarter choice of relays. This gain diminishes in the high SINR region, since the interference generated from the cognitive system 


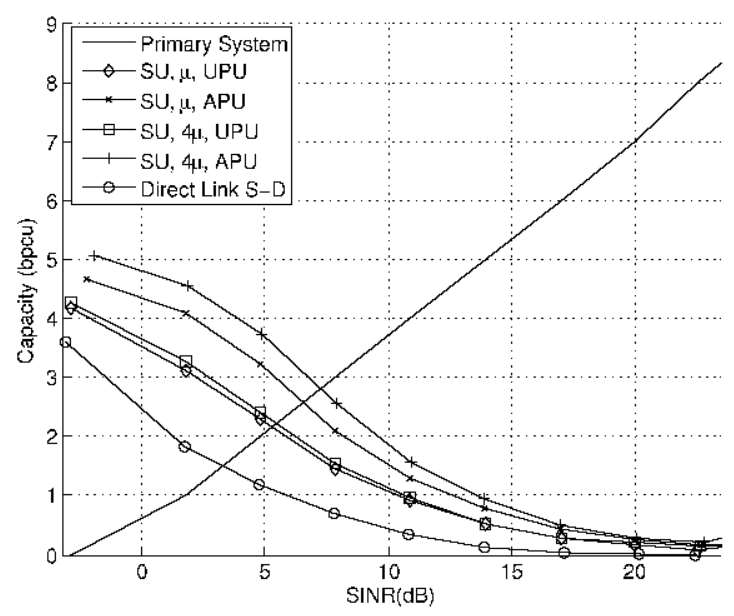

Fig. 2. Performance trade-offs in cognitive networks. UPU and APU denote unaware PU and aware PU, respectively. Relay densily is $\mu$ and SU denotes cognitive user.

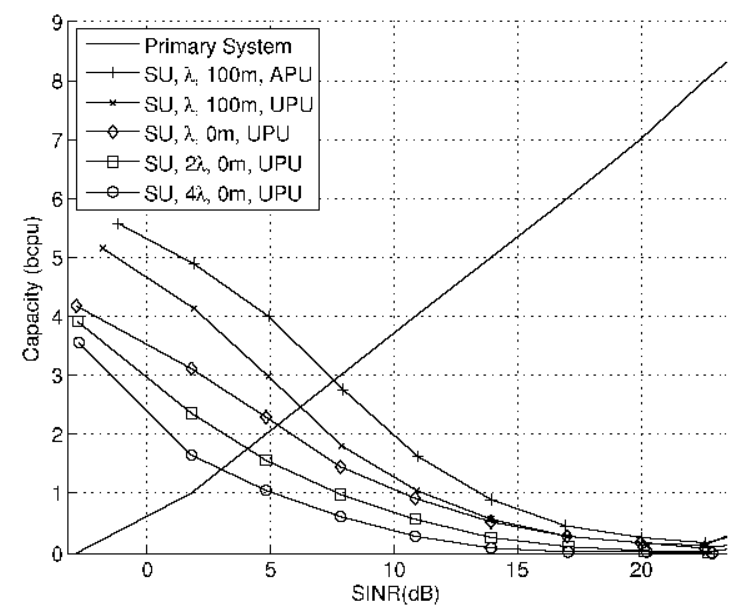

Fig. 3. Performance trade-offs in cognitive networks. $\lambda$ is PU density and the distance $0 \mathrm{~m}, 100 \mathrm{~m}$ corresponds to guard zone.

has small impact. Increasing the number of relays yields negligible gain when PUs are oblivious to the existence of the SUs. On the other hand, when the primary system is interference-aware, it can actually benefit from a larger number of available relays, for similar reasons as before. If we assume best case scenario, the aggregate gain, compared to the direct S-D link rate, is significant. Based on these results we conclude that the system mainly benefits if we can make its terminals smarter, implying that relay selection has a vital role in performance.

The effect of the remaining parameters is illustrated in Fig. 3. Intuitively, as the PU density increases the probability that a primary user will be located on the boundary of the guard zone, increases as well. Therefore system performance will be limited by the worst case user but, from a practical point of view, we are able to serve more users. Reversely, larger protection zone leaves less room for placing PUs, under the premise that we should always put a cognitive unit somewhere in the cell. Though, it is casily seen that capacity gain is significant, if an area of radius $100 \mathrm{~m}$ around each PU is kept interference free.

Note here, that although most curves depict SU perlormance, we interpret the results from a PU perspective. Essentially the conclusions on $\mathrm{PU}$ performance stem from the dual interpretation of the $\mathrm{SU}$ rates. If we fix a value $R_{\mathrm{SU}}$ for the SU rate, then for each SU case we can pinpoint on the PU curve, the respective rates that the primary system achieves at the same SINR value.

To sum up, in this paper we studied performance trade-olls in cognitive networks. Various aspects of these systems were investigated and the eflect of different parameters was evaluated. The analysis, performed here, can be extended to include more network parameters and architectures and capture more practical cases. Our results are a positive indication that cognitive networks are feasible and could be part of future generation wireless networks.

\section{REFERENCES}

[1] S. Avestimchr, S. N. Diggavi, and D. Tsc, "Wircless network information flow: A deterministic approach," submitted to IEEE Trans. Information Theory, preprint available at http://arxiv.org/abs/0906.5394.

[2] —, "Wireless network information flow," in Proc. Allerton Conf. on Comm., Contr. and Comp., Sep. 2007.

「31 A. Amaudruz and C. Fragouli, "Combinatorial algorithms for wireless information flow," in Proc. ACM-SIAM Symp. Discrete. Algorithms, 2009.

41 S. Avestimehr, S. N. Diggavi, and D. Tse, "Approximate capacity of Gaussian relay networks," in IEEE Int. Symp. of Inform. Theory, Jul. 2008.

[5] N. Schrammar and M. Skoglund, "Uncoded transmission in wireless relay networks using deterministic modeling," in Proc. Asilomar Conf. SSC, 2009.

[6] M. Anand and P. R. Kumar, "On approximating Gaussian relay networks by deterministic networks," in Proc. Inform. Theory Workshop (ITW), Oct. 2009. 